\title{
Group Exercise versus Personal Training for Prostate Cancer Patients: A Pilot Randomized Trial*
}

\author{
Daniel Santa Mina ${ }^{1,2,3 \#}$, Paul Ritvo ${ }^{2,4}$, Andrew G. Matthew ${ }^{1}$, Ajay Rampersad ${ }^{3}$, Helga Stein ${ }^{1}$, \\ Angela M. Cheung ${ }^{5,6}$, John Trachtenberg ${ }^{1,5,6}$, Shabbir M. H. Alibhai ${ }^{5,6}$ \\ ${ }^{1}$ The Department of Surgical Oncology, Princess Margaret Hospital, Toronto, Canada; ${ }^{2}$ School of Kinesiology and Health Science, \\ York University, Toronto, Canada; ${ }^{3}$ Kinesiology Program, University of Guelph-Humber, Toronto, Canada; ${ }^{4}$ Cancer Care Ontario, \\ Toronto, Canada; ${ }^{5}$ University Health Network, Toronto, Canada; ${ }^{6}$ University of Toronto, Toronto, Canada. \\ Email: \#pritvo@yorku.ca, andrew.matthew@uhn.ca, aj.ramp@gmail.com, helga_stein@yahoo.com.br, \\ \{angela.cheung, john.trachtenberg, shabbir.alibhai\}@uhn.ca
}

Received January 22 ${ }^{\text {nd }}, 2012$; revised February 20 ${ }^{\text {th }}, 2012$; accepted March $14^{\text {th }}, 2012$

\begin{abstract}
Background: Previous studies have demonstrated psychosocial and physical benefit from exercise for older men treated with androgen deprivation therapy (ADT) for prostate cancer (PCa). To date, different intervention delivery methods have not been assessed. This study compared the effect of group-based exercise (GBE) to personal training (PT) in men undergoing ADT for PCa. Methods: 13 participants (mean age $=68.2$, SD $=7.2$ ) were randomly assigned to GBE or PT for 8 weeks. Participants exercised for 60 minutes three times per week at a moderate-vigorous intensity. Results: 10 participants completed the intervention. At post-intervention, the PT group improved: fatigue, systolic BP, waist circumference, body fat percentage, and maximal leg strength; participants in the GBE group improved leg strength. At 8 weeks, maximal upper body strength in the PT group was greater than the GBE group. There were no adverse events associated with moderate-high intensity training in this population of older men. Conclusion: PT may be more efficacious than GBE in improving several physical fitness outcomes and fatigue in men with PCa who are androgen deprived. Due to the small sample size and attrition, these results require cautious interpretation and confirmation from adequately powered trials.
\end{abstract}

Keywords: Group Exercise; Personal Training; Prostate Cancer; Pilot Randomized Trial

\section{Introduction}

In Canadian men, prostate cancer (PCa) is the most common form of cancer and the third leading cause of cancer-related death [1]. Fortunately, advances in detection and treatment have led to earlier diagnosis and treatment, improving 10-year survival rates to over $95 \%$ [2-5]. Thus, the population of PCa survivors is growing. A common treatment for men with advanced PCa is androgen deprivation therapy (ADT) which may be used for several years or indefinitely [6,7]. Unfortunately, ADT leads to numerous undesirable consequences that negatively affect physical function and health-related quality of life (HRQOL), including: reduced lean mass and bone density, increased fat mass, and anemia, that

\footnotetext{
*Funding: This study was funded by the University of Guelph- Humber Research Grant Fund Program and the Princess Margaret Hospital Foundation.

Conflict of Interest: The authors declare that there are no conflicts of interest in the conducting of this trial or preparation of the manuscript.

${ }^{\#}$ Corresponding author.
}

collectively contribute to physical weakness and fatigue [8-11]. In response to these prevalent and often distressing side effects, exercise has been proposed and examined as a complimentary therapeutic modality to ADT given its physical, physiological, and psychosocial benefits [12-17].

Recent systematic reviews have examined the role of exercise in PCa, documenting numerous physical and psychosocial benefits and no exacerbation of disease status (no increase in prostate-specific antigen or testosterone) [18-20]. Specifically for PCa patients receiving ADT, six published trials have examined the role of exercise on physical fitness and HRQOL outcomes [16,17, 21-24]. Most of these trials have employed hospitalbased, supervised exercise training programs which have yielded more robust benefits for participants compared to home-based, unsupervised exercise training. While both exercise delivery methods have inherent strengths, they each have weaknesses that limit patient participation and adherence, possibly limiting overall intervention efficacy and chronic exercise behaviour adoption. Hospital-based, 
one-on-one exercise training is costly and has poor generalizability to community-based programs that lack equivalent resources and oncologic expertise. Additionally, distance to exercise facility, associations between hospital and difficult treatments, and travel costs related to urban tertiary care centres are reported barriers to hospital-based exercise adherence and participation [25-29]. Conversely, home-based programs are limited by the lack of supervision and tangible professional support required to ensure full compliance with, and safety of, the exercise prescription. Failure to adequately comply and progress with the exercise prescription may result in failure to stimulate physiologic adaptation and performance improvements. To address the limitations of hospital-based, supervised training and home-based exercise programs, supervised group-based exercise (GBE) may be an ideal alternative mode of exercise program delivery for cancer patients. GBE can provide participants with qualified supervision and motivation, immediate feedback and instruction, as well as social support, while significantly reducing the personnel and facility-requirement costs as multiple participants can be trained in a fraction of the time it takes to deliver personal training for the same group.

Culos-Reed and colleagues conducted two trials of home-based exercise for PCa patients using supplemental 90-minute weekly GBE classes (called "Booster Sessions") to facilitate program adherence through social support and education $[16,17]$. Although these sessions were well attended ( $\sim 80 \%$ attendance rate), the homebased intervention demonstrated limited effectiveness at improving fatigue and other fitness outcomes. The lack of improvement, compared to other facility-based trials in this population, may be attributable to a lack of compliance with the specific exercise prescription in the home-based setting. These studies did demonstrate, however, that patients are willing to attend GBE classes, and if conducted on a more frequent basis (2 - 3 times per week), may be more efficacious than home-based exercise with minimal increases in cost. Furthermore, the GBE format which facilitates social support and provides trainer supervision and motivation may improve participation rates and adherence. To date, exercise interventions for cancer patients that are principally GBE have not been directly compared to equivalent personal training (PT) programs which are generally considered the gold-standard for exercise training. This type of investigation is warranted given the presumed resource-utilization discrepancy between intervention delivery models, with PT likely being considerably more costly. Moreover, no study has examined exercise in the same cohort across two geographically unique sites. Information regarding attendance to urban and suburban locations is useful to address various local facilitators and barriers to exercise participation in cancer populations [30-32]. The objectives of this pilot trial are to assess the feasibility and efficacy of 8 weeks of GBE versus PT at two training locations on fatigue and HRQOL in men receiving ADT for PCa.

\section{Methods}

\subsection{Trial Design}

This pilot study was a prospective, two-arm randomized pilot trial of GBE versus PT. The exercise interventions were delivered in the Greater Toronto Area in Ontario, Canada, at an urban tertiary care hospital and a suburban university. Trainers, assessors, and participants were not blinded to group allocation. Concealed randomization was performed using sequentially numbered opaque envelopes containing group assignments provided to participants following the baseline assessment. This study was approved by the research ethics review committees at the participating institutions.

\subsection{Recruitment}

Eligible patients were approached for participation by a research team member following a urology clinic appointment in June 2011. Patients could also respond to study information posters in the clinic waiting areas. Eligible participants were informed of the study procedures, and potential benefits before providing written informed consent. Participants were paid twenty dollars per week if they attended at least two exercise sessions in that week to defray travel-related costs.

\subsection{Participants}

Patients were eligible for the study inclusion if they: 1) had histologically confirmed PCa; 2) were currently receiving ADT or recently completed ADT (within the past 3 months); 3) had non-metastatic, asymptomatic disease; 4) were between ages 45 and 75 years; and 5) did not have a comorbidity that would preclude participation in a moderate-vigorous intensity exercise program (including: severe coronary artery disease (Canadian Cardiovascular Society class III); uncontrolled hypertension (BP > 200/100) or significant congestive heart failure (New York Heart Association class III); uncontrolled pain; neurological or musculoskeletal ailment inhibiting exercise; high risk of fracture according to the World Health Organization Fracture Risk Assessment Tool (WHO FRAX); and diagnosed psychotic, addictive, or major cognitive disorders).

\subsection{Exercise Training Interventions}

The exercise interventions were delivered at two sites on 
alternating weeks (i.e. weeks $1,3,5$, and 7 at the hospital and weeks 2, 4, 6, and 8 at the university). The urban/hospital-based training was conducted in a cancer survivorship facility at the hospital. This facility was equipped with a group-exercise studio, resistance bands, aerobic steps, free weights, resistance bands, stability balls and medicine balls. The exercise facility at the university was equipped with a group-exercise studio, aerobic exercise machines (elliptical machine, treadmill, and stationary cycles), plate-loaded weight-training machines, and free weights.

Three senior kinesiology undergraduate students with personal training certification and group exercise experience delivered the intervention under the supervision of certified exercise physiologists. All exercise sessions were 60 minutes in duration, offered three times per week for eight weeks. (Due to a civic holiday, one training day was lost, resulting in a maximum training volume of 23 sessions over 8 weeks). Participants were encouraged by their trainers to exercise at $70 \%-85 \%$ of their measured maximum heart rate for aerobic exercise and 6 - 12 repetition maximum for resistance training. Heart rate was measured throughout GBE and PT sessions using a heart rate monitor or oxygen saturation monitor.

Although there are inherent differences in program delivery between GBE and PT, equivalence between the GBE and PT groups was attempted by using similar fitness parameters: 5 minute warm-up, 25 minutes of aerobic exercise, 25 minutes of resistance training, and a 5 minute cool-down. For all participants, resistance training included one exercise for the upper and lower extremities, chest, back, and core muscles per training session. Each participant also received an exercise manual with important details about the exercise program including: safety precautions, location and traveling directions for the exercise sessions, an exercise log, and a calendar detailing the dates, times, and locations of their GBE or PT sessions.

The only difference between the intervention groups was the format in which the exercise program was delivered (GBE or PT). GBE classes employed low-impact, aerobic exercise routines and basic resistance training using free-weights, calisthenic exercises, and/or resistance bands and stability balls. PT sessions employed standard aerobic training equipment or trainer-led lowimpact step exercises and resistance training using plateloaded machine, resistance bands, or free-weight exercises (location-dependent).

\subsection{Assessments}

Each participant completed assessments at baseline and post-intervention (8 weeks). All assessments were conducted at the university by a certified exercise physiologist with assistance from senior kinesiology undergraduate students.

\subsection{Outcome Measures}

Our primary outcome measure was feasibility and was assessed by the participation rate, adherence to the exercise intervention (as indicated by session attendance), attrition, and adverse events related to exercise testing or training. Patient satisfaction surveys were also administered at the end of the trial to obtain information that might further guide the development of a large-scale randomized trial. Participants were asked to rate how much they agreed with a statement regarding their participation $(1=$ strongly disagree to $5=$ strongly agree $)$. Secondary outcomes were incorporated to examine the effectiveness of GBE and PT on psychosocial and physical measures.

\subsubsection{Demographic, Disease, and Treatment Information}

Demographic (e.g. age, educational status, etc.), disease (tumour stage and Gleason score), and treatment (ADT regimen) information were collected at baseline.

\subsubsection{Psychosocial Outcomes}

Cancer-specific fatigue was assessed using the 13-item Functional Assessment of Cancer Therapy-Fatigue (FACT-F) [33]. The FACT-F has high test-retest reliability $(r=0.87)$, excellent internal consistency (Cronbach's $\alpha=0.93-0.95$ ), and good convergent/discriminant validity [33,34]. The FACT-F has been previously used in trials examining the effect of exercise in men with PCa $[21,35]$. The minimal clinically important difference (MCID) for the FACT-F is 3.5 points. PCa-specific HRQOL was examined using two measures: the Functional Assessment of Cancer Treatment-Prostate (FACT-P) [36] and the Patient-Oriented Prostate Utility Scale (PORPUS) [37]. The FACT-P a widely used instrument that combines 12 items for assessing PCa-specific issues with 35 items of the generic FACT (FACT-G) scale to produce a 47 -item scale. The FACT-G is an extensively validated cancer-specific measure with high reliability and internal consistency estimates in multiple languages [36,38-42]. The FACT-P has been validated in older cancer patients [41] and was able to detect differences in PCa patients related to exercise intervention in 3 previous studies $[12,35,43]$. The MCID for the FACT-P is 5.5 points. The PORPUS is a psychometric and utility HRQOL scale that has found to be reliable in discriminating between known groups of PCa patients as well as 
change over time in known groups [44]. The PORPUS is a 10-item questionnaire that asks patients to describe their HRQOL status by selecting 1 of 5 possible conditions in the following domains: pain and disturbing body sensations, energy, support from family and friends, communication with doctor, emotional well-being, urinary frequency, bladder control, sexual function, sexual drive, and bowel problems. The PORPUS has been used in several studies to assess treatment-related changes in HRQOL [45-49].

\subsubsection{Physical Outcomes}

Aerobic fitness was assessed by direct measurement of peak oxygen consumption $\left(\mathrm{VO}_{2}\right.$ peak) using the modified Bruce treadmill protocol $[50,51]$. Grip strength was assessed according to the Canadian Society for Exercise Physiology protocol [52]. To examine strength increases of the upper and lower extremities, the Brzycki method [53] was employed to determine 1-repetition maximum (1RM) for the plate-loaded seated bench press and leg press. This method of estimating 1RM is recommended for older adults and has been used previously in cancer populations [54,55]. Balance was assessed using the Functional-Reach Test that is intended for an elderly population and measures the distance that a patient can safely reach forward without taking a step [56,57]. Participant height and weight were recorded and used to calculate body mass index (BMI). Waist circumference was measured according to the World Health Organization guidelines [50]. Body density and body fat percentage were calculated using sum of three skinfolds (chest, abdomen, and thigh) [50,58]. BMD $\left(\mathrm{g} / \mathrm{cm}^{2}\right)$ was assessed using quantitative ultrasound (QUS) of the calcaneus. Calcaneal QUS is commonly used to estimate BMD and requires patients to place their heel between two probes that transmit pulses ultrasound waves [59,60]. Correlations between QUS and the gold-standard, dual X-ray absorptiometry (DXA), at the same anatomical sites have been found to be as high as $r=0.8-0.9$ [61] and was shown to predict fracture risk independent of DXA [62].

\subsection{Statistical Analysis}

Data were double-entered by two research staff to ensure accuracy. Baseline comparisons were performed using independent-samples t-tests for continuous variables and chi-squared analyses for categorical variables. Analysis of covariance was conducted to compare differences in psychosocial and physical outcomes at post-intervention, controlling for the baseline value of the outcome of interest. Per protocol analyses were conducted on participants that completed baseline and post-intervention assessments. Data were analyzed using the Statistical Package for Social Sciences version 19.0.

\section{Results}

\subsection{Participant Characteristics and Feasibility Assessment}

We recruited 13 (24\%) of the 54 eligible participants we approached. Eligible participants cited the following reasons for non-participation: no time or not available during the intervention period $(\mathrm{n}=12)$, too far to travel for exercise $(n=3)$, not feeling well enough to participate $(n=1)$, not interested or already exercising $(n=12)$, or no reason provided $(n=13)$. The 13 participants were randomly assigned to receive GBE $(n=6)$ or PT $(n=7)$ for 8 weeks. Participant characteristics are presented in Tables 1 and 2. There were no significant differences between groups at baseline. Three participants from the GBE group did not complete the trial: one participant experienced symptomatic metastatic disease and was withdrawn from the study after 1 week; two other participants indicated that the exercise facility was too far to commute on a regular basis and withdrew from the study after the baseline assessment. Study non-completers were more likely to be smokers than completers (chi-square = 10.0; $\mathrm{p}=0.019$ ), and non-completers were more likely to be in the GBE group (chi-square $=4.55, \mathrm{p}=0.033$ ). There were no other differences between study completers and non-completers (data not shown).

The mean attendance rates for PT and GBE sessions among completers were $91 \%(\mathrm{SD}=8.0)$ and $88 \%(\mathrm{SD}=$ 10.9), respectively $(p=0.645)$. Training sessions (combined PT and GBE) were attended at the urban location $94 \%$ of the time versus $83 \%$ of the time at suburban location $(p=0.582)$. In the patient satisfaction survey, $100 \%$ of participants agreed or strongly agreed with the statements: "I felt that participating in the exercise program was a positive experience and helped to enhance my quality of life", and "I will continue to exercise after this program is over." Seventy percent of participants agreed or strongly agreed with the statement "I feel that I have the confidence to continue working out on my own after this program ends”. Forty-four percent of participants agreed or strongly agreed with the statement "I prefer to work out at the university rather than the hospital because traveling to the university was easier", while $33 \%$ strongly disagreed with this statement. Sixty percent disagreed or strongly disagreed with the statement "I prefer to work out in a hospital-based exercise facility". Finally, $60 \%$ of participants strongly disagreed with the statement "I would prefer to do group-based exercise rather than personal training” with the PT group being more likely to strongly disagree than the GBE group (p = 0.019). No other between-group differences were found in the patient satisfaction survey.

There were no adverse events related to the exercise intervention. 
Table 1. Baseline characteristics of continuous variables.

\begin{tabular}{|c|c|c|c|}
\hline Variable & PT $(n=7)$ & GBE $(n=6)$ & P-value \\
\hline Age (years) & $66.3(8.6)$ & $70.5(4.9)$ & 0.311 \\
\hline \multicolumn{4}{|c|}{ Psychosocial Outcomes } \\
\hline QOL-PORPUS & $60.9(11.5)$ & $58.9(14.6)$ & 0.982 \\
\hline QOL-FACT-P & $107.7(21.4)$ & $111.6(29.1)$ & 0.788 \\
\hline Fatigue-FACT-F & $35.0(10.2)$ & $38.0(10.5)$ & 0.614 \\
\hline \multicolumn{4}{|c|}{ Physical Outcomes } \\
\hline Weight (kg) & $86.5(8.7)$ & $85.3(19.0)$ & 0.887 \\
\hline $\mathrm{WC}(\mathrm{cm})$ & $106.5(7.1)$ & $102.8(8.5)$ & 0.403 \\
\hline BMI $\left(\mathrm{kg} / \mathrm{m}^{2}\right)$ & $29.2(3.2)$ & $27.7(3.5)$ & 0.429 \\
\hline Body Fat \% & $34.6(3.2)$ & $33.1(9.4)$ & 0.699 \\
\hline Peak $\mathrm{VO}_{2}\left(\mathrm{LO}_{2} / \mathrm{min}\right)$ & $2.7(0.6)$ & $2.5(0.4)$ & 0.349 \\
\hline Peak $\mathrm{VO}_{2}\left(\mathrm{mLO}_{2} / \mathrm{kg} / \mathrm{min}\right)$ & $31.8(8.2)$ & $29.4(5.7)$ & 0.554 \\
\hline 1RM-bench press (kg) & $53.2(36.2)$ & 33.5 (19.9) & 0.254 \\
\hline 1RM-Leg press (kg) & $122.5(54.7)$ & $104.6(62.7)$ & 0.593 \\
\hline Grip Strength (kg) & $74(14.2)$ & $70.7(18.8)$ & 0.728 \\
\hline Functional Reach (cm) & $36.4(8.1)$ & $32.3(8.7)$ & 0.397 \\
\hline Resting Heart Rate (bpm) & $72.5(19.8)$ & $74.2(21.0)$ & 0.890 \\
\hline Resting Systolic Blood Pressure (mmHg) & $138.6(18.6)$ & $128.0(15.4)$ & 0.294 \\
\hline $\mathrm{BMD} \mathrm{g} / \mathrm{cm}^{2}$ & $0.49(0.08)$ & $0.52(0.08)$ & 0.463 \\
\hline
\end{tabular}

Data are presented as Mean (SD); p-value for between-group differences using independent sample t-test.

Table 2. Baseline characteristics of categorical variables.

\begin{tabular}{|c|c|c|c|}
\hline Variable & PT $(n=7)$ & GBE $(n=6)$ & p-value \\
\hline Caucasian & $5(71)$ & $4(57)$ & 0.563 \\
\hline Married & $6(86)$ & $3(50)$ & 0.401 \\
\hline Post-High School Education & $6(86)$ & $3(50)$ & 0.353 \\
\hline Working (part-time or full-time) & $5(71)$ & $2(33)$ & 0.246 \\
\hline Currently Smoking (yes) & $1(14)$ & $2(33)$ & 0.321 \\
\hline \multicolumn{4}{|l|}{ Gleason Score } \\
\hline 7 & $4(57)$ & $4(67)$ & \multirow{3}{*}{0.401} \\
\hline $8-10$ & $3(43)$ & $0(0)$ & \\
\hline Not Available & $0(0)$ & $1(17)$ & \\
\hline \multicolumn{4}{|l|}{ ADT Indication } \\
\hline Biochemical Relapse & $4(57)$ & $2(33)$ & \multirow{3}{*}{0.672} \\
\hline Adjuvant to Radiation & $1(14)$ & $1(17)$ & \\
\hline Metastatic Disease & $2(29)$ & $3(50)$ & \\
\hline \multicolumn{4}{|l|}{ Treatment } \\
\hline Currently on LHRH Alone & $5(71)$ & $4(67)$ & \multirow{3}{*}{0.503} \\
\hline Currently on LHRH + Anti-Androgen & $1(14)$ & $0(0)$ & \\
\hline Discontinued ADT in Past 3 Months & $1(14)$ & $2(33)$ & \\
\hline
\end{tabular}

Data are presented as Frequency (\% of group); p-value for chi-square; LHRH = luteinizing hormone-releasing hormone, ADT = androgen deprivation therapy. 


\subsection{Psychosocial Outcomes}

Within and between-group results of psychosocial outcomes are presented in Table 3. From baseline to postintervention, there was a clinically important improvement in fatigue for the PT group that trended towards significance $(p=0.09)$. There were no other withingroups improvements for either the PT or GBE groups. There were no between-group differences at post-intervention for fatigue or HRQOL.

\subsection{Physical Outcomes}

Within and between-group results of the physical outcomes are presented in Table 4. The PT group demon- strated improvements in resting systolic blood pressure ( $\mathrm{p}=0.033)$, body fat percentage $(\mathrm{p}=0.001)$, and maximal lower body strength $(p=0.002)$ from baseline to post-intervention follow-up. There was also a trend towards improved waist circumference in this group over the 8-week intervention $(\mathrm{p}=0.076)$. The GBE group demonstrated borderline statistically significant improvements in maximal upper body $(p=0.054)$ and lower body ( $p=0.076)$ strength. After 8 weeks of training, the PT group demonstrated significantly greater improvement in maximal lower body strength than the GBE group $(\mathrm{p}=0.038)$. Conversely, the GBE group demonstrated greater improvement in maximal upper body strength after the 8-week intervention $(p=0.013)$.

Table 3. Fatigue and quality of life after 8 weeks of exercise training.

\begin{tabular}{ccccccc}
\hline \multirow{2}{*}{ Variable } & \multicolumn{2}{c}{ Baseline } & \multicolumn{2}{c}{ 8 Weeks } & \multirow{2}{*}{ F } & P-Value (ANCOVA) \\
\cline { 2 - 5 } & PT & GBE & PT & GBE & & \\
\hline FACT-F & $35.0(3.9)$ & $36.0(5.7)$ & $40.7(3.5)^{*}$ & $38.3(7.6)$ & 0.463 & 0.518 \\
FACT-P & $107.7(8.1)$ & $108.3(16.3)$ & $120.0(8.1)$ & $103(18.0)$ & 2.106 & 0.190 \\
PORPUS & $74.0(14.2)$ & $59.2(6.6)$ & $72.7(6.1)$ & $67.1(6.6)$ & 0.000 & 0.995 \\
\hline
\end{tabular}

Per protocol analysis (PT: $n=7$; GBE: $n=3$ ); Data are presented as mean (standard error); Between-groups analysis: p-value reported for ANCOVA when adjusting for baseline value; Within-groups analysis: *significant change from baseline $(\mathrm{p}<0.1)$; Scales oriented to indicate improvement in well-being with increased score.

Table 4. Physical fitness after 8 weeks of exercise training.

\begin{tabular}{|c|c|c|c|c|c|c|}
\hline \multirow{2}{*}{ Variable } & \multicolumn{2}{|c|}{ Baseline } & \multicolumn{2}{|c|}{8 Weeks } & \multicolumn{2}{|c|}{ Between-Groups Difference } \\
\hline & PT & GBE & PT & GBE & $\mathbf{F}$ & P-Value (ANCOVA) \\
\hline Resting Heart Rate (bpm) & $72.6(7.5)$ & $81.7(13.9)$ & $74.0(5.1)$ & $83.7(18.2)$ & 0.1 & 0.760 \\
\hline Resting Systolic Blood Pressure (mmHg) & $138.6(7.0)$ & $118.7(8.7)$ & $124.0(10.2) \dagger$ & $109.3(18.1)$ & 0.421 & 0.537 \\
\hline Weight (kg) & $86.5(3.3)$ & $72.3(2.5)$ & 86.9 (2.9) & $72.4(1.0)$ & 3.11 & 0.121 \\
\hline Body Mass Index $\left(\mathrm{kg} / \mathrm{m}^{2}\right)$ & $29.2(1.2)$ & 26.6(0.7) & $29.1(2.7)$ & $26.4(0.2)$ & 2.88 & 0.133 \\
\hline Waist Circumference (cm) & $106.5(2.7)$ & $98.5(2.8)$ & $104.6(7.5)^{*}$ & $97.3(0.6)$ & 0.000 & 1.000 \\
\hline Body Fat \% & $34.6(1.2)$ & $32.3(4.1)$ & $28.0(4.1) \ddagger$ & $28.6(3.2)$ & 0.245 & 0.636 \\
\hline Bone Mineral Density $\left(\mathrm{g} / \mathrm{cm}^{2}\right)$ & $0.49(0.03)$ & $0.56(0.053)$ & $0.52(0.11)$ & $0.58(0.077)$ & 0.009 & 0.928 \\
\hline Functional Reach Distance (cm) & $36.4(3.1)$ & $27.9(2.2)$ & $36.8(3.0)$ & $33.0(4.1)$ & 0.000 & 0.992 \\
\hline Bench Press 1RM (kg) & $53.2(13.7)$ & $32.3(2.0)$ & $54.0(3.0)$ & $37.7(2.8)^{*}$ & 10.94 & 0.013 \\
\hline Leg Press 1RM (kg) & $136.4(44.5)$ & $98.2(8.4)$ & $259.9(55.5) \ddagger$ & $136.7(18.0)^{*}$ & 6.999 & 0.038 \\
\hline Peak $\mathrm{VO}_{2}\left(\mathrm{LO}_{2} / \mathrm{min}\right)$ & $2.7(0.21)$ & $2.23(0.27)$ & $2.7(0.27)$ & $2.25(0.18)$ & 0.034 & 0.860 \\
\hline Peak $\mathrm{VO}_{2}\left(\mathrm{mLO}_{2} / \mathrm{kg} / \mathrm{min}\right)$ & $31.8(3.1)$ & $30.7(3.4)$ & $33.1(3.5)$ & $31.2(2.7)$ & 0.124 & 0.735 \\
\hline Grip Strength (kg) & $74.0(5.4)$ & $63.7(6.2)$ & $72.7(6.1)$ & $53.7(6.5)$ & 0.000 & 0.995 \\
\hline
\end{tabular}

Per protocol analysis (PT: $n=7$; GBE: $n=3$ ); Data are presented as mean (standard error); Between-groups analysis: p-value reported for ANCOVA when adjusting for baseline value; Within-groups analysis: *significant change from baseline ( $\mathrm{p}<0.1)$; †significant change from baseline ( $<<0.05)$; $\ddagger$ significant change from baseline $(\mathrm{p}<0.01)$. 


\section{Discussion}

Our study demonstrated that men with PCa are able to safely participate in a moderate-vigorous intensity exercise program, across two sites (urban and suburban) with similar program compliance. We also show that our recruitment to the PT versus GBE trial resembles the recruitment rate in previous exercise studies in PCa [21,24, 35], and that participants are largely satisfied with participation in such a trial. Participant satisfaction is also reflected in the high adherence rate in both intervention groups, however it appears that PT may be more preferable, as indicated by greater attrition in the GBE group and participant feedback. Our results are statistically underpowered to make conclusive statements about outcome measurements. However, our between-groups findings suggest that participants in our PT and GBE interventions experienced similar benefits in terms of most health outcomes, with the exception of greater improvements in lower body strength for the PT group while the GBE group demonstrated greater improvements in upper body strength. This latter finding may be due to a floor effect in the GBE group, although between-group differences at baseline were not statistically significant. Within-group improvements were observed for PT participants in terms of fatigue, blood pressure, body fat percentage, waist circumference, and upper body strength, while only upper and lower body strength improved for the GBE group. There was also a notable increase of twice the MCID for HRQOL using the FACT-P for the PT group but these findings were not statistically significant and require further examination in a larger trial.

Several trials have assessed GBE or supervised exercise for cancer patients, but to our knowledge this is the first study to explicitly examine the feasibility and effect of PT versus GBE in a sample of cancer patients. Previous studies in men with PCa have examined supervised, facility-based exercise [12-14,35,43] and home-based exercise with and without weekly booster sessions [16, 63,64], with facility-based trials generally providing more and larger benefits for participants. The results of this trial suggest that PCa survivors equally attend PT and GBE sessions, as well as urban and suburban exercise facilities. This indicated that location and delivery mode of exercise may not be a significant barrier to exercise in this group. This is contrary to our a priori hypothesis that exercise in the suburban/university setting would be preferable to participants due to less traffic, easier access to parking, and less association between the exercise facility and difficult cancer treatments. This is important for cancer-exercise program designers considering various venues for program delivery. Furthermore, 2 participants in the GBE group withdrew from the study citing travel distance as a barrier. Whether or not this is a spurious finding related to GBE needs to examined in larger trials.

The preference for exercise intervention delivery models has been widely examine in recent years. While cancer patients commonly cite home-based, unsupervised exercise programs as most preferable [30,65-68], there are several issues with this program design. First, supervised exercise is most amenable to monitoring adverse events and exercise technique that is important to prevent injuries. Second, supervision permits the exercise instructor to immediately respond to the motivational climate of the exercise participants, increasing or decreasing the intensity of the program accordingly. Third, there is likely a social determinant of routine exercise behaviour in a supervised setting that is lacking in a homebased setting, as participants become committed or feelresponsible to their training staff and possibly their fellow GBE participants. In contrast, supervised exercise requires training staff and a facility that is likely an institutional barrier to program implementation. As well, supervised training programs (unless home-based) require the participants to travel and incur associated costs, which is a reported barrier to routine exercise [25-28]. Given that failing to maintain exercise behaviours is often associated with a return of side effects $[17,69]$, cancer-exercise programmers must develop programs that are clinically effective and amenable to sustained participation. An ideal program may be one that includes initial facility-based supervision to ensure appropriate exercise technique and safety monitoring, followed by a home-based exercise program with routine follow-ups that allow for exercise adaptation and progression. Our current study suggests that GBE and PT interventions can routinely be attended by PCa patients. And, while it appears that PT may be somewhat more beneficial than GBE and especially appropriate for high-needs patients that require a high degree of supervision, it is likely also more costly which may prevent its common integration into cancer-exercise programs. Future research into the cost-effectiveness of cancer-exercise programs is notably absent and greatly needed.

Strengths of this study include a randomized design, supervised exercise training, as well as low study attrition and high program adherence. An additional strength is the use of several physical outcomes to assess changes in physical fitness that are known to be negatively impacted by ADT, such as musculoskeletal strength, cardiorespiratory fitness, fracture risk (balance and BMD), and body composition. Furthermore, the participants unanimously endorsed the exercise program, however our remuneration for participation in this trial may skew participant interpretation. On an open-ended question regarding comments on the exercise program, one par- 
ticipant said "I would like to continue in a program like this, it gave me a lot of energy", and another said "[the exercise program] gave me a very positive perspective on my physical condition and what I need to do to keep that up”.

There are several limitations in this study that require cautious interpretation of this study and will need to be addressed in future assessments of GBE versus PT in cancer patients. As a pilot trial, the small sample was appropriate to assess the feasibility but inadequate to draw conclusions regarding intervention effect. Compounding the small sample size were 3 dropouts in the GBE group that severely limit the between-group comparisons. Based on the effect size required to detect a MCID in the FACT-F as the primary outcome, 39 participants per group would be required for analysis in a randomized controlled trial (three-armed). Other limitations of this study are: short duration of the exercise intervention, no long-term follow-up, and no blinding of fitness assessors. Despite our efforts, it is difficult to be sure that the intensity of the intervention is the same between the PT and GBE groups, and conceivable that personal trainers may unconsciously train their participants more intensely because they were more able to respond to fatigue and effort cues. Specifically, it was challenging to ensure that the resistance training portion of the interventions was equal between the PT and GBE groups because in the GBE setting, increases in repetitions or load to meet the exercise prescription were not adapted until the subsequent exercise class after feedback from the participant was received. In an effort to standardize training intensity between the intervention arms so that the training effect could be attributed to GBE versus PT (rather than intensity), we continually measured heart rate throughout the exercise sessions, ensuring that it was maintained within the exercise prescription, and adapted the resistance training portions as quickly as was feasible. In the future, it would be prudent to instruct participants in GBE to increase their resistance training intensity (load) throughout their exercise session by keeping a variety of weights/bands convenient to use as necessary.

Including our study, participation rates in exercise studies with PCa survivors ranges from 22\% to $86 \%$ (mean $=47 \%$ ), with comparable participation rates in home-based and facility-based trials (46\% and 48\%, respectively). Data regarding the number of cancer survivors meeting physical activity recommendations is mixed [70]; however, it appears that PCa patients specifically are somewhat disinterested or not able to participate in exercise interventions. Our study offered free-of-charge fitness testing and training as well as travel cost compensation, yet only $24 \%$ of those invited ended up participating. Future studies should examine the various motivational factors and barriers associated with exercise in this population and design programs that would facilitate program participation.

\section{Conclusion}

Our trial demonstrates that PT and GBE in alternating exercise locations can be feasibly conducted in a population of older men with PCa receiving ADT. In the interest of cost-savings, GBE may be preferable for institutions considering the implementation of a cancer-exercise program given comparable efficacy to PT in several health outcomes. However, our findings do suggest that more, and greater benefits may be possible with PT when compared to GBE. Interpretation of the intervention effect results of this pilot trial should be guarded given the small sample size and disproportionate attrition between groups. A larger, randomized trial is needed to confirm our findings.

\section{Acknowledgements}

We would like to acknowledge the various contributions of the following individuals: Dr. Elaine Popp, Dr. George Bragues, Michael O’Leary, Denise Gardner, Brent Welsh, Joseph Gibbons, Tara McDougall, William Hilton, Zameer Tharani, Dr. Luba Slatkovska, and the staff at the Electronic Living Laboratory for Cancer Survivorship Research (ELLICSR), in particular, Dr. David Wiljer, Dr. Sara Urowitz, Dr. Jennifer Jones, and Dr. Pamela Catton.

\section{REFERENCES}

[1] Canadian Cancer Society's Steering Committee on Cancer Statistics, “Canadian Cancer Statistics 2011,” Canadian Cancer Society, Toronto, 2011.

[2] A. Bill-Axelson, L. Holmberg, F. Filen, M. Ruutu, H. Garmo, C. Busch, et al., "Radical Prostatectomy versus Watchful Waiting in Localized Prostate Cancer: The Scandinavian Prostate Cancer Group-4 Randomized Trial," Journal of the National Cancer Institute, Vol. 100, No. 16, 2008, pp. 1144-1154. doi:10.1093/jnci/djn255

[3] A. L. Potosky, J. Legler, P. C. Albertsen, J. L. Stanford, F. D. Gilliland, A. S. Hamilton, et al., "Health Outcomes after Prostatectomy or Radiotherapy for Prostate Cancer: Results from the Prostate Cancer Outcomes Study,” Journal of the National Cancer Institute, Vol. 92, No. 19, 2000, pp. 1582-1592. doi:10.1093/jnci/92.19.1582

[4] M. Quinn and P. Babb, "Patterns and Trends in Prostate Cancer Incidence, Survival, Prevalence and Mortality. Part I: International Comparisons," British Journal of Urology International, Vol. 90, No. 2, 2002, pp. 162-173. doi:10.1046/j.1464-410X.2002.02823.X

[5] P. J. Saylor, N. L. Keating and M. R. Smith, "Prostate Cancer Survivorship: Prevention and Treatment of the Adverse Effects of Androgen Deprivation Therapy," $J$ Gen Intern Med, Vol. 24, Suppl. 2, 2009, pp. S389- S394.

[6] M. R. Cooperberg, G. D. Grossfeld, D. P. Lubeck and P. 
R. Carroll, "National Practice Patterns and Time Trends in Androgen Ablation for Localized Prostate Cancer," Journal of the National Cancer Institute, Vol. 95, No. 13, 2003, pp. 981-989. doi:10.1093/jnci/95.13.981

[7] V. B. Shahinian, Y. F. Kuo, J. L. Freeman, E. Orihuela and J. S. Goodwin, "Increasing Use of GonadotropinReleasing Hormone Agonists for the Treatment of Localized Prostate Carcinoma," Cancer, Vol. 103, No. 8, 2005, pp. 1615-1624. doi:10.1002/cncr.20955

[8] S. M. Alibhai, S. Gogov and Z. Allibhai, "Long-Term Side Effects of Androgen Deprivation Therapy in Men with Non-Metastatic Prostate Cancer: A Systematic Literature Review," Critical Reviews in Oncology and Hematology, Vol. 60, No. 3, 2006, pp. 201-215. doi:10.1016/j.critrevonc.2006.06.006

[9] J. A. Smith, Jr., M. S. Soloway and M. J. Young, “Complications of Advanced Prostate Cancer," Urology, Vol. 54, Suppl. 6A, 1999, pp. 8-14.

[10] M. R. Smith, J. S. Finkelstein, F. J. McGovern, A. L. Zietman, M. A. Fallon, D. A. Schoenfeld, et al., "Changes in Body Composition During Androgen Deprivation Therapy for Prostate Cancer,” The Journal of Clinical Endocrinology and Metabolism, Vol. 87, No. 2, 2002, pp. 599-603. doi:10.1210/jc.87.2.599

[11] D. A. Galvao, N. A. Spry, D. R. Taaffe, R. U. Newton, J. Stanley, T. Shannon, et al., "Changes in Muscle, Fat and Bone Mass after 36 Weeks of Maximal Androgen Blockade for Prostate Cancer,” BJU International, Vol. 102, No. 1, 2008, pp. 44-47. doi:10.1111/j.1464-410X.2008.07539.x

[12] R. J. Segal, R. D. Reid, K. S. Courneya, S. C. Malone, M. B. Parliament, C. G. Scott, et al., "Resistance Exercise in Men Receiving Androgen Deprivation Therapy for Prostate Cancer,” Journal of Clinical Oncology, Vol. 21, No. 9, 2003, pp. 1653-1659. doi:10.1200/JCO.2003.09.534

[13] D. A. Galvao, K. Nosaka, D. R. Taaffe, N. Spry, L. J. Kristjanson, M. R. McGuigan, et al., "Resistance Training and Reduction of Treatment Side Effects in Prostate Cancer Patients," Medicine and Science in Sports and Exercise, Vol. 38, No. 12, 2006, pp. 2045-2052. doi:10.1249/01.mss.0000233803.48691.8b

[14] D. A. Galvao, N. Spry, D. R. Taaffe, J. Denham, D. Joseph, D. S. Lamb, et al., "A Randomized Controlled Trial of an Exercise Intervention Targeting Cardiovascular and Metabolic Risk Factors for Prostate Cancer Patients from the Radar Trial," BMC Cancer, Vol. 9, 2009, pp. 419426.

[15] C. L. Carmack Taylor, C. Demoor, M. A. Smith, A. L. Dunn, K. Basen-Engquist, I. Nielsen, et al., "Active for Life after Cancer: A Randomized Trial Examining a Lifestyle Physical Activity Program for Prostate Cancer Patients," Psycho-Oncology, Vol. 15, No. 10, 2006, pp. 847-862. doi:10.1002/pon.1023

[16] S. N. Culos-Reed, J. L. Robinson, H. Lau, K. O’Connor and M. R. Keats, "Benefits of a Physical Activity Intervention for Men with Prostate Cancer," Journal of Sport and Exercise Psychology, Vol. 29, No. 1, 2007, pp. 118127.
[17] S. N. Culos-Reed, J. Robinson, H. Lau, L. Stephenson, M. Keats, S. Norris, et al., "Physical Activity for Men Receiving Androgen Deprivation Therapy for Prostate Cancer: Benefits from a 16-Week Intervention,” Supportive Care in Cancer, Vol. 18, No. 5, 2010, pp. 591-599. doi:10.1007/s00520-009-0694-3

[18] J. W. Keogh and R. D. Macleod, "Body Composition, Physical Fitness, Functional Performance, Quality of Life, and Fatigue Benefits of Exercise for Prostate Cancer Patients: A Systematic Review," Journal of Pain and Symptom Management, Vol. 43, No. 1, 2011, pp. 96-110.

[19] L. Thorsen, K. S. Courneya, C. Stevinson and S. D. Fossa, "A Systematic Review of Physical Activity in Prostate Cancer Survivors: Outcomes, Prevalence, and Determinants,” Supportive Care in Cancer, Vol. 16, No. 9, 2008, pp. 987-997. doi:10.1007/s00520-008-0411-7

[20] D. Santa Mina, P. Ritvo, R. Segal, S. N. Culos-Reed and S. M. H. Alibhai. "Exercise after Prostate Cancer Diagnosis,” In: J. Saxton and A. Daley, Eds., Exercise and Cancer Survivorship: Impact on Health Outcomes and Quality of Life, Springer, New York, 2010.

[21] R. J. Segal, R. D. Reid, K. S. Courneya, S. C. Malone, M. B. Parliament, C. G. Scott, et al., "Resistance Exercise in Men Receiving Androgen Deprivation Therapy for Prostate Cancer," Journal of Clinical Oncology, Vol. 21, No. 9, 2003, pp. 1653-1659. doi:10.1200/JCO.2003.09.534

[22] D. A. Galvao, D. R. Taaffe, N. Spry, D. Joseph and R. U. Newton, "Combined Resistance and Aerobic Exercise Program Reverses Muscle Loss in Men Undergoing Androgen Suppression Therapy for Prostate Cancer without Bone Metastases: A Randomized Controlled Trial," Journal of Clinical Oncology, Vol. 28, No. 2, 2010, pp. 340-347. doi:10.1200/JCO.2009.23.2488

[23] D. A. Galvao, K. Nosaka, D. R. Taaffe, N. Spry, L. J. Kristjanson, M. R. McGuigan, et al., "Resistance Training and Reduction of Treatment Side Effects in Prostate Cancer Patients," Medicine and Science in Sports and Exercise, Vol. 38, No. 12, 2006, pp. 2045-2052. doi:10.1249/01.mss.0000233803.48691.8b

[24] C. L. Carmack Taylor, C. Demoor, M. A. Smith, A. L. Dunn, K. Basen-Engquist, I. Nielsen, et al., “Active for Life after Cancer: A Randomized Trial Examining a Lifestyle Physical Activity Program for Prostate Cancer Patients," Psycho-Oncology, Vol. 15, No. 10, 2006, pp. 847862. doi:10.1002/pon.1023

[25] C. Peeters, A. Stewart, R. Segal, E. Wouterloot, C. G. Scott and T. Aubry, "Evaluation of a Cancer Exercise Program: Patient and Physician Beliefs," Psycho-Oncology, Vol. 18, No. 8, 2009, pp. 898-902. doi:10.1002/pon.1406

[26] C. Stevinson and K. R. Fox, "Feasibility of an Exercise Rehabilitation Programme for Cancer Patients," European journal of cancer care, Vol. 15, No. 4, 2006, pp. 386-396. doi:10.1111/j.1365-2354.2006.00677.x

[27] M. Craike, P. Livingston and M. Botti, “An Exploratory Study of the Factors That Influence Physical Activity for Prostate Cancer Survivors,” Supportive Care in Cancer, 
Vol. 19, No. 7, 2011, pp. 1019-1028. doi:10.1007/s00520-010-0929-3

[28] R. Segal, W. Evans, D. Johnson, J. Smith, S. Colletta, J. Gayton, et al., "Structured Exercise Improves Physical Functioning in Women with Stages I and II Breast Cancer: Results of a Randomized Controlled Trial," Journal of Clinical Oncology, Vol. 19, No. 3, 2001, pp. 657-665.

[29] W. Demark-Wahnefried, "Print-to-Practice: Designing Tailored Print Materials to Improve Cancer Survivors' Dietary and Exercise Practices in the Fresh Start Trial," Nutrition Today, Vol. 42, No. 3, 2007, pp. 131-138. doi:10.1097/01.NT.0000277790.03666.95

[30] L. W. Jones and K. S. Courneya, "Exercise Counseling and Programming Preferences of Cancer Survivors," Cancer Practice, Vol. 10, No. 4, 2002, pp. 208-215. doi:10.1046/j.1523-5394.2002.104003.x

[31] L. Q. Rogers, S. J. Markwell, K. S. Courneya, E. McAuley and S. Verhulst, "Exercise Preference Patterns, Resources, and Environment among Rural Breast Cancer Survivors," Journal of Rural Health, Vol. 25, No. 4, 2009, pp. 388-391. doi:10.1111/j.1748-0361.2009.00249.x

[32] L. Q. Rogers, S. J. Markwell, S. Verhulst, E. McAuley and K. S. Courneya, "Rural Breast Cancer Survivors: Exercise Preferences and Their Determinants," PsychoOncology, Vol. 18, No. 4, 2009, pp. 412-421. doi:10.1002/pon.1497

[33] D. Cella, "The Functional Assessment of Cancer Therapy-Anemia (Fact-an) Scale: A New Tool for the Assessmente of Outcomes in Cancer Anemia and Fatigue," Seminars in Hematology, Vol. 34, No. 3, 1997, pp. 13-19.

[34] S. B. Yellen, D. F. Cella, K. A. Webster, C. Blendowski and E. Kaplan, "Measuring Fatigue and Other Anemia-Related Symptoms with the Functional Assessment of Cancer Therapy (Fact) Measurement System,” Journal of Pain and Symptom Management, Vol. 13, 1997, pp. 63-74.

[35] R. J. Segal, R. D. Reid, K. S. Courneya, R. J. Sigal, G. P. Kenny, D. G. Prud'Homme, et al., "Randomized Controlled Trial of Resistance or Aerobic Exercise in Men Receiving Radiation Therapy for Prostate Cancer," Journal of Clinical Oncology,” Vol. 27, No. 3, 2009, pp. 344351. doi:10.1200/JCO.2007.15.4963

[36] P. Esper, F. Mo, G. Chodak, M. Sinner, D. Cella and K. J. Pienta, "Measuring Quality of Life in Men with Prostate Cancer Using the Functional Assessment of TherapyProstate Instrument," Urology, Vol. 50, No. 6, 1997, pp. 920-928. doi:10.1016/S0090-4295(97)00459-7

[37] M. Krahn, P. Ritvo, J. Irvine, G. Tomlinson, A. Bezjak, J. Trachtenberg, et al., "Construction of the Patient-Oriented Prostate Utility Scale (PORPUS): A Multiattribute Health State Classification System for Prostate Cancer," Journal of Clinical Epidemiology, Vol. 53, No. 9, 2000, pp. 920-930. doi:10.1016/S0895-4356(00)00211-0

[38] P. S. Brucker, K. Yost, J. Cashy, K. Webster and D. Cella, "General Population and Cancer Patient Norms for the Functional Assessment of Cancer Therapy-General (FACT-G)," Evaluations And The Health Professions, Vol. 28, No. 2, 2005, pp. 192-211. doi:10.1177/0163278705275341
[39] D. Cella, L. Hernandez, A. E. Bonomi, M. Corona, M. Vaquero, G. Shiomoto, et al., "Spanish Language Translation and Initial Validation of the Functional Assessment of Cancer Therapy Quality-of-Life Instrument,” Medical Care, Vol. 36, No. 9, 1998, pp. 1407-1418. doi:10.1097/00005650-199809000-00012

[40] H. Fumimoto, K. Kobayashi, C. H. Chang, S. Eremenco, Y. Fujiki, S. Uemura, et al., "Cross-Cultural Validation of an International Questionnaire, the General Measure of the Functional Assessment of Cancer Therapy Scale (FACT-G), for Japanese,” Quality of Life Research, Vol. 10, No. 8, 2001, pp. 701-709. doi:10.1023/A:1013851216181

[41] J. Overcash, M. Extermann, J. Parr, J. Perry and L. Balducci, "Validity and Reliability of the Fact-G Scale for Use in the Older Person with Cancer," American Journal of Clinical Oncology, Vol. 24, No. 6, 2001, pp. 591-596. doi:10.1097/00000421-200112000-00013

[42] P. Winstead-Fry and A. Schultz, "Psychometric Analysis of the Functional Assessment of Cancer Therapy-General (Fact-G) Scale in a Rural Sample,” Cancer, Vol. 79, No. 12, 1997, pp. 2446-2452.

doi:10.1002/(SICI)1097-0142(19970615)79:12<2446::AI D-CNCR23>3.0.CO;2-Q

[43] U. Monga, S. L. Garber, J. Thornby, C. Vallbona, A. Kerrigan, T. N. Monga, et al., "Exercise Prevents Fatigue and Improves Quality of Life in Prostate Cancer Patients Undergoing Radiotherapy," Archives of Physical Medicine and Rehabilitation, Vol. 88, No. 11, 2007, pp. 14161422. doi:10.1016/j.apmr.2007.08.110

[44] P. Ritvo, J. Irvine, G. Naglie, G. Tomlinson, A. Bezjak, A. Matthew, et al., "Reliability and Validity of the Porpus, a Combined Psychometric and Utility-Based Quality-ofLife Instrument for Prostate Cancer,” Journal of Clinical Epidemiology, Vol. 58, 2005, pp. 466-474.

[45] M. D. Krahn, K. E. Bremner, G. Tomlinson and G. Naglie, "Utility and Health-Related Quality of Life in Prostate Cancer Patients 12 Months after Radical Prostatectomy or Radiation Therapy," Prostate Cancer and Prostatic Diseases, Vol. 12, No. 4, 2009, pp. 361-368. doi:10.1038/pcan.2009.32

[46] P. Toren, S. M. H. Alibhai, A. Matthew, M. Nesbitt, R. Kalnin, N. Fleshner, et al., "The Effect of Nerve-Sparing Surgery on Patient-Reported Continence Post-Radical Prostatectomy," Canadian Urology Association Journal, Vol. 3, No. 6, 2009, pp. 465-470.

[47] J. Trachtenberg, A. Bogaards, R. A. Weersink, M. A. Haider, A. Evans, S. A. McCluskey, et al., "Vascular Targeted Photodynamic Therapy with Palladium-Bacteriopheophorbide Photosensitizer for Recurrent Prostate Cancer Following Definitive Radiation Therapy: Assessment of Safety and Treatment Response," Journal of Urology, Vol. 178, No. 5, 2007, pp. 1974-1979. doi:10.1016/j.juro.2007.07.036

[48] J. Trachtenberg, R. A. Weersink, S. R. Davidson, M. A. Haider, A. Bogaards, M. R. Gertner, et al., "VascularTargeted Photodynamic Therapy (Padoporfin, Wst09) for Recurrent Prostate Cancer after Failure of External Beam Radiotherapy: A Study of Escalating Light Doses,” BJU 
International, Vol. 102, No. 5, 2008, pp. 556-562. doi:10.1111/j.1464-410X.2008.07753.X

[49] D. Santa Mina, A. Matthew, J. Trachtenberg, G. Tomlinson, C. L. Guglietti, S. M. H. Alibhai, et al., "Physical Activity and Quality of Life after Radical Prostatectomy," Canadian Urology Association Journal, Vol. 4, No. 3, 2010, pp. 180-186.

[50] V. H. Heyward, "Advanced Fitness Assessment and Exercise Prescription,” Human Kinetics, Windsor, 2002.

[51] J. Lerman, R. A. Bruce, E. Sivarajan, G. M. Pettet and S. Trimble, "Low Level Dynamic Exercises for Earlier Cardiac Rehabilitation: Aerobic and Hemodynamic Responses," Archives of Physical Medicine and Rehabilitation, Vol. 57, No. 1976, pp. 355-360.

[52] Canadian Society for Exercise Physiology, Fitness Assessment and Interpretation, "The Canadian Physical Activity Fitness and Lifestyle Approach,” Ottawa, 2004. pp. 7.1-7.57.

[53] M. Brzycki, "Strength Testing-Predicting One-Repetition Max from Reps-to-Fatigue," Journal of Physical Education, Recreation \& Dance, Vol. 64, No. 1, 1993, pp. 8890.

[54] I. C. Backer, E. Van Breda, A. Vreugdenhil, M. R. Nijziel, A. D. Kester and G. Schep, "High-Intensity Strength Training Improves Quality of Life in Cancer Survivors,” Acta Oncologica, 2007, pp. 1143-1151.

[55] L. Adamsen, M. Quist, J. Midtgaard, C. Andersen, T. Moller, L. Knutsen, et al., "The Effect of a Multidimensional Exercise Intervention on Physical Capacity, WellBeing and Quality of Life in Cancer Patients Undergoing Chemotherapy," Supportive Care in Cancer, Vol. 14, No. 2, 2006, pp. 116-127. doi:10.1007/s00520-005-0864-x

[56] P. W. Duncan, D. K. Weiner, J. Chandler and S. Studenski, "Functional Reach: A New Clinical Measure of Balance,” Journal of Gerontology, Vol. 45, No. 6, 1990, pp. M192-M197.

[57] D. K. Weiner, P. W. Duncan, J. Chandler and S. A. Studenski, "Functional Reach: A Marker of Physical Frailty," Journal of the American Geriatric Society, Vol. 40, No. 3, 1992, pp. 203-207.

[58] A. S. Jackson and M. L. Pollock, "Generalized Equations for Predicting Body Density of Men,” British Journal of Nutrition, Vol. 40, No. 3, 1978, pp. 497-504. doi:10.1079/BJN19780152

[59] G. Guglielmi, G. Scalzo, F. de Terlizzi and W. C. Peh, "Quantitative Ultrasound in Osteoporosis and Bone Metabolism Pathologies," Radiologic Clinics of North America, Vol. 48, No. 3, 2010, pp. 577-588. doi:10.1016/j.rcl.2010.02.013

[60] M. A. Krieg, R. Barkmann, S. Gonnelli, A. Stewart, D. C. Bauer, L. Del Rio Barquero, et al., "Quantitative Ultrasound in the Management of Osteoporosis: The 2007 Iscd Official Positions,” Journal of Clinical Densitometry, Vol.
11, No. 1, 2008, pp. 163-187. doi:10.1016/j.jocd.2007.12.011

[61] E. M. Lewiecki, B. Richmond and P. D. Miller, "Uses and Misuses of Quantitative Ultrasonography in Managing Osteoporosis," Cleveland Clinic Journal of Medicine, Vol. 73, No. 8, 2006, pp. 742-746. doi:10.3949/ccjm.73.8.742

[62] A. Moayyeri, S. Kaptoge, N. Dalzell, S. Bingham, R. N. Luben, N. J. Wareham, et al., "Is Qus or Dxa Better for Predicting the 10-Year Absolute Risk of Fracture?," Journal of Bone Mineral Research, Vol. 24, No. 7, 2009, pp. 1319-1325. doi:10.1359/jbmr.090212

[63] S. N. Culos-Reed, J. W. Robinson, H. Lau, L. Stephenson, M. Keats, S. Norris, et al., "Physical Activity for Men Receiving Androgen Deprivation Therapy for Prostate Cancer: Benefits from a 16-Week Intervention,” Supportive Care in Cancer, Vol. 18, No. 5, 2009, pp. 591-599.

[64] P. M. Windsor, K. F. Nicol and J. Potter, “A Randomized, Controlled Trial of Aerobic Exercise for Treatment-Related Fatigue in Men Receiving Radical External Beam Radiotherapy for Localized Prostate Carcinoma," Cancer, Vol. 101, No. 3, 2004, pp. 550-557. doi:10.1002/cncr.20378

[65] C. Stevinson, V. Capstick, A. Schepansky, K. Tonkin, J. K. Vallance, A. B. Ladha, et al., "Physical Activity Preferences of Ovarian Cancer Survivors,” Psycho-Oncology, Vol. 18, No. 4, 2009, pp. 422-428. doi:10.1002/pon.1396

[66] L. Q. Rogers, K. S. Courneya, S. Verhulst, S. J. Markwell and E. McAuley, "Factors Associated with Exercise Counseling and Program Preferences among Breast Cancer Survivors," Journal of Physical Activity and Health, Vol. 5, No. 5, 2008, pp. 688-705.

[67] L. W. Jones, B. Guill, S. T. Keir, K. Carter, H. S. Friedman, D. D. Bigner, et al., "Exercise Interest and Preferences among Patients Diagnosed with Primary Brain Cancer," Supportive Care in Cancer, Vol. 15, No. 1, 2007, pp. 47-55. doi:10.1007/s00520-006-0096-8

[68] J. K. Vallance, K. S. Courneya, L. W. Jones and T. Reiman, "Exercise Preferences among a Population-Based Sample of Non-Hodgkin's Lymphoma Survivors," European Journal of Cancer Care (England), Vol. 15, No. 1, 2006, pp. 34-43. doi:10.1111/j.1365-2354.2005.00617.x

[69] K. S. Courneya, C. M. Friedenreich, H. A. Quinney, A. L. Fields, L. W. Jones and A. S. Fairey, "A Randomized Trial of Exercise and Quality of Life in Colorectal Cancer Survivors," European Journal of Cancer Care, Vol. 12, No. 4, 2003, pp. 347-357. doi:10.1046/j.1365-2354.2003.00437.x

[70] W. Demark-Wahnefried, N. M. Aziz, J. H. Rowland and B. M. Pinto, "Riding the Crest of the Teachable Moment: Promoting Long-Term Health after the Diagnosis of Cancer,” Journal of Clinical Oncology, Vol. 23, No. 24, 2005, pp. 5814-5830. doi:10.1200/JCO.2005.01.230 